\title{
Determinantes del Diagnóstico Periodontal
}

\author{
Determinants of Periodontal Diagnosis
}

\author{
Botero JE ${ }^{1}$, Bedoya $\mathrm{E}^{1}$
}

\section{RESUMEN}

Un correcto diagnóstico periodontal es necesario para la realización de una terapia periodontal exitosa en nuestro paciente. Entendiendo que la enfermedad periodontal es un proceso infeccioso-inflamatorio, diferentes variables se deben analizar clínicamente para determinar el diagnóstico. El diagnóstico entonces es un análisis concienzudo de la expresión clínica de la enfermedad, desde gingivitis hasta periodontitis. Este artículo analiza los determinantes más importantes del diagnóstico periodontal utilizados en la práctica clínica diaria.

Rev. Clin. Periodoncia Implantol. Rehabil. Oral Vol. 3(2); 94-99, 2010.

Palabras clave: Profundidad al sondaje, nivel de inserción clínica, pérdida ósea, sangrado al sondaje, periodontitis crónica, periodontitis agresiva.

\section{ABSTRACT}

The correct diagnosis of periodontal disease is a pre-requisite for an appropriate periodontal treatment. Periodontal disease is an infectious-inflammatory process that affects different clinical variables that must be analyzed before reaching the diagnosis. The diagnosis should be a carefully performed analysis of the clinical expression of the disease, from gingivitis to periodontitis. This article reviews the most important determinants of periodontal diagnosis.

Rev. Clin. Periodoncia Implantol. Rehabil. Oral Vol. 3(2); 94-99, 2010.

Key words: Probing depth, clinical attachment level, bone loss, bleeding on probing, chronic periodontitis, aggressive periodontitis.

\section{INTRODUCCIÓN}

La enfermedad periodontal es considerada una enfermedad infecciosa-inflamatoria, que de acuerdo al grado de compromiso puede llevar a la pérdida total de los tejidos de soporte del diente. Considerando que la etiología de la enfermedad es principalmente infecciosa (placa bacteriana), el tratamiento se enfoca fundamentalmente en el control de la infección y reducción de la inflamación. Por lo tanto, para ejecutar un tratamiento periodontal exitoso es necesario determinar de forma adecuada el diagnóstico y pronóstico periodontal. En este documento se analizan y se plantean los determinantes más importantes que son empleados para emitir un diagnóstico periodontal.

\section{Etiopatogénesis de la Enfermedad Periodonta}

Todo comienza cuando las bacterias producen factores de virulencia (Ej.: lipopolisacarido-LPS, acido lipoteicoico) y estos entran en contacto con las células del epitelio del surco pero es en especial atención, las células del epitelio de unión (EU) las que producen defensinas y citoquinas pro-inflamatorias ${ }^{(1)}$. Las defensinas son péptidos antimicrobianos que dañan la superficie de las bacterias, permitiendo su eliminación. Pero son de gran importancia la producción de IL-1 y TNF $\alpha$, generando cambios a nivel vascular. Incrementan el calibre de los vasos sanguíneos e inducen la expresión de proteínas de adhesión celular. Adicionalmente, producen IL8, una citoquina con actividad quimiotáctica para PMNs. De esta forma, los PMNs son atraídos al sitio donde se acumulan las bacterias, salen de los vasos sanguíneos y se acumulan en el tejido conectivo adyacente al surco alterando el tejido conectivo adyacente al EU. Muchos PMNs se abren paso por los espacios intercelulares del EU y salen al surco donde se degranulan ${ }^{(2)}$, liberando consigo reactivos del oxígeno (ROls) y enzimas como catepsina G, lactoferrina, defensinas, mieloperoxidasa, metaloproteinasas (MMP-8) y serin proteasas ${ }^{(3,4)}$. Si bien todos estos reactivos biológicos son nocivos para las bacterias, también lo pueden ser para los tejidos periodontales y algún daño tisular microscópico puede esperarse. No obstante, el agente infeccioso es controlado en la mayoría de casos, el estímulo disminuye y se establece un balance de la respuesta inmune.

Después de estimulada la respuesta inmune innata, desencadena la respuesta inmune adaptativa y aparecen en el tejido conectivo linfocitos T CD4 y linfocitos $B$, ayudando a resolver el proceso inflamatorio ${ }^{(5)}$. La estimulación de linfocitos toma entre 5 y 7 días en alcanzar su mayor activación. Por lo tanto, una buena respuesta innata es fundamental para mantener la salud periodontal. Los linfocitos T CD4 producen citoquinas (IFN $\gamma, \mathrm{IL}-2$ ) que promueven una mejor actividad de macrófagos y co-estimulan a los linfocitos $\mathrm{B}$ a producir anticuerpos tipo IgG e IgA neutralizantes( ${ }^{(6)}$. El resultado es una respuesta inmune que controla los microorganismos que se están acumulando en el surco periodontal, de forma silenciosa y sin expresar signos clínicos inflamatorios evidentes a simple vista. A medida que progresa el proceso inflamatorio éste se vuelve crónico y comienza la degradación de los tejidos de soporte, dando como resultado la formación de la bolsa periodontal, pérdida de inserción clínica y pérdida ósea.

\section{PARÁMETROS CLÍNICOS PERIODONTALES}

\section{Profundidad Sondeable (PS)}

Cabe recordar que el espacio que se forma alrededor de los dientes, entre la encía y la superficie radicular, representa nuestro punto principal de análisis. Este espacio puede ser considerado un "surco" o una "bolsa periodontal". Aunque estudios en animales demostraron que este espacio en ausencia total de placa bacteriana no existía ${ }^{(7)}$, en los humanos siempre estará presente y por lo tanto su medición ha sido tema de debate.

Para hablar de profundidad sondeable es necesario analizar cuidadosamente la unidad de medida que utilizamos y existe una limitación importante al medir el espacio entre la encía y el diente, y es que los espacios se miden como área o por el volumen que pueden ocupar. Pero este no es el caso del espacio del surco periodontal, ya que utilizamos una medida lineal en un solo plano y tomado en seis sitios de los dientes. Aún así, debe ser calculada cuidadosamente en milímetros, tomando como referencia el margen gingival, que en la mayoría de casos coincide con la línea amelocementaria (CEJ) o ligeramente coronal a esta. Cuando el margen esta apical a la CEJ, se denomina una recesión de tejido marginal y este es uno de los resultados de la pérdida de inserción (Figura 1).

Como la determinación de la posición del margen gingival es dependiente de un punto de referencia fijo (CEJ), es necesario definir una nueva referencia cuando esta ha desaparecido. Es preciso consignar cuál fue el punto de referencia nuevo, sea una restauración, el margen de una corona o incluso desde borde oclusal. En recesiones vestibulares que involucran abfracciones es posible trazar una línea imaginaria desde las superficies proximales (Figura 2). 


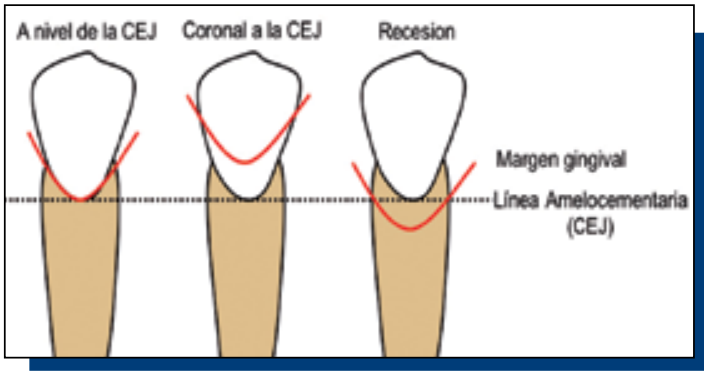

Figura 1. Esquema representativo de la posición del margen gingival en relación a la línea amelocementaria (CEJ).

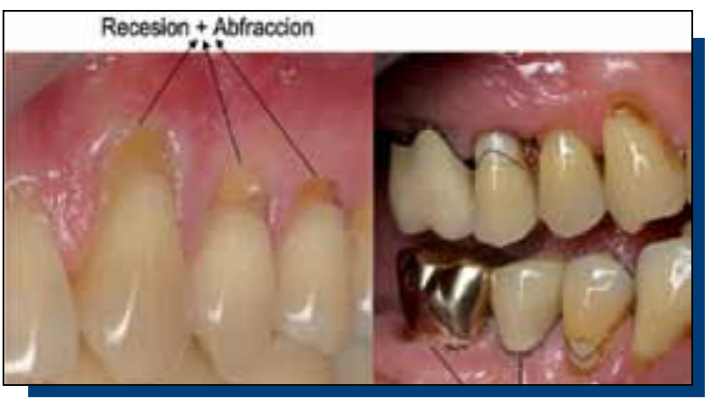

Figura 2. Imagen fotográfica que muestra casos en donde es necesario identificar un punto de referencia para medir el margen gingival.

El surco periodontal se define como el espacio alrededor de los dientes entre la encía marginal y la superficie del diente y que esta limitado en su parte más apical por las células más coronales del epitelio de unión $(E U)^{(8)}$. Se ha considerado en estudios clínicos en humanos que este espacio puede medir entre 1 y $3 \mathrm{~mm}$ en ausencia de inflamación clínica $^{(9-12)}$. No obstante, en estudios histológicos la distancia desde las células más coronales del EU hasta el margen gingival mide entre 0.69 y $1 \mathrm{~mm}^{(13,14)}$. Esto sugiere que durante el sondaje hay un desprendimiento de la adherencia de las células del EU, sin llegar hasta el tejido conectivo. Pero para efectos clínicos prácticos, un surco periodontal no presenta sangrando al sondaje y puede medir hasta $3.9 \mathrm{~mm}$ (Figura 3).

En contraste, la bolsa periodontal se define como la profundización patológica del surco periodontal, dada por la pérdida ósea y de inserción periodontal ${ }^{(8,15)}$. Aunque el límite de $4 \mathrm{~mm}$ parezca arbitrario, se ha observado que frecuentemente se asocia con sitios que presentan inflamación tanto histológica como clínica y ya se observa pérdida ósea radiográfica. Medidas superiores a $4 \mathrm{~mm}$ resultan más evidentes con signos claros de destrucción periodontal ${ }^{(16)}$. Esta transición de un surco a una bolsa periodontal representa uno de los signos cardinales de la periodontitis, dado que es producida por la pérdida de inserción. Para efectos clínicos prácticos, una bolsa periodontal puede ser considerada a partir de $4 \mathrm{~mm}$ y deben presentar sangrado al sondaje, pérdida de inserción y pérdida ósea radiográfica (Figuras 3 y 4 ).

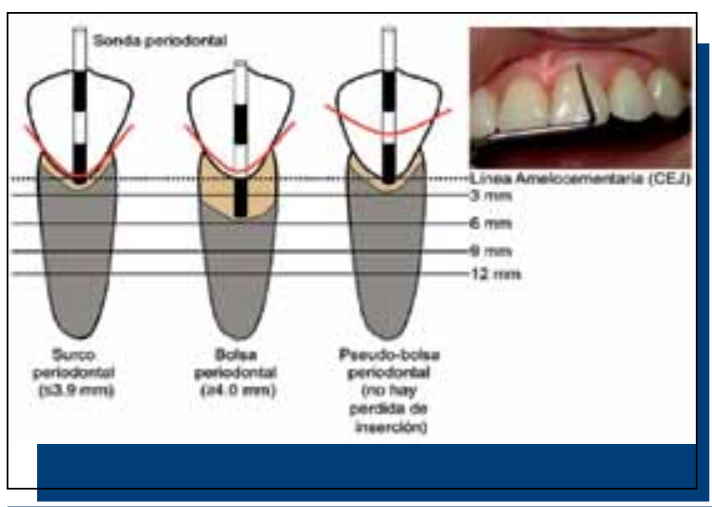

Figura 3. Esquema representativo de las diferentes relaciones que existen entre la profundidad sondeable y la pérdida de inserción. La zona sombreada gris representa el nivel de inserción remanente (incluye inserción de tejido conectivo gingival y ligamento periodontal).

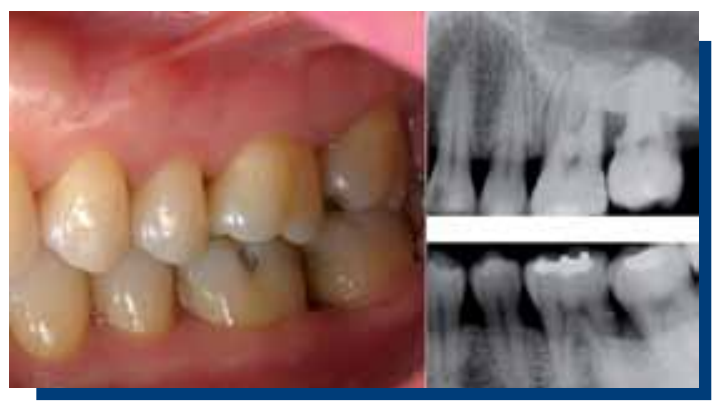

Figura 4. Aspecto clínico y radiográfico de la periodontitis. Nótese la pérdida ósea radiográfica en zona distal de 26 y 36 .

Pero podemos encontrarnos con casos en donde exista una profundidad al sondaje incrementada en ausencia de pérdida de inserción y pérdida ósea. Esto es por que el punto de referencia para esta medida es el margen gingival, y este puede variar en su dimensión dependiendo de grado de inflamación o agrandamiento gingival.

Con el desarrollo del edema gingival o engrosamiento de la encía marginal (agrandamiento gingival), el margen se desplaza en sentido coronal a la línea amelocementaria. A este hallazgo se le denomina "pseudo bolsa periodontal" y aunque no hay pérdida de soporte periodontal, puede acumular altos niveles de placa bacteriana subgingival y con el tiempo desarrollar destrucción periodontal (Figura 3). Se considera que sitios que presenten PS residual después de la terapia periodontal pueden tener más riesgo de progresión de la enfermedad, evidenciado con un OR 7.7 (odds ratio) para profundidades de $5 \mathrm{~mm}$ y OR 9.3 para profundidades de $6 \mathrm{~mm}^{(17)}$. Es así como la profundidad sondeable (PS) se puede interpretar de tres maneras posibles dependiendo de la forma como se presente y esto es fundamental para el diagnóstico periodontal.

\section{Nivel de Inserción Clínica (NIC)}

Esta medida hace referencia a las fibras de tejido conectivo gingivales que se insertan al cemento radicular a través de fibras de Sharpey. Al igual que la medida de PS, es una medida lineal más que un área de soporte periodontal, tal cual y como ocurre naturalmente. A diferencia de las fibras del ligamento, la inserción de la encía se da de forma constante a $1.07 \mathrm{~mm}$ (aproximadamente) coronal a la cresta ósea. Sin embargo, en algunos casos nos encontramos dientes que tienen una inserción de tejido conectivo supracrestal mucho más largo y por lo tanto una reducción en el nivel óseo sin que esto indique que sean más susceptibles a mayor pérdida de inserción ${ }^{(18)}$. Pero esto debe ser analizado cuidadosamente. Un estudio clínico ${ }^{(11)}$ mostró que el ancho biológico podía variar en sujetos con periodontitis y a veces se encontraban sitios que mostraban pérdida ósea importante pero con una profundidad al sondaje no tan incrementada que no coincidía con lo que podía denominarse el nivel más apical de la pérdida ósea. Esta variación puede ser explicada por variables individuales en la inflamación periodontal y metabolismo de los tejidos periodontales. Es posible que en algunos sitios se pierda altura ósea a una tasa diferente a la del tejido conectivo, resultando en una distancia de tejido conectivo mayor $(4.16 \mathrm{~mm}+/-1.32 \mathrm{~mm})$.

Más coronal a la inserción de TC de la encía, se encuentra el epitelio de unión $(0.97 \mathrm{~mm})$. Por lo tanto, si sumamos la medida del TC y EU nos da aproximadamente $2 \mathrm{~mm}$ (Ancho Biológico) ${ }^{(13)}$, y esta es la distancia a la que frecuentemente se observa la cresta ósea desde la CEJ. Apical a la cresta ósea se continúa el ligamento periodontal rodeando la raíz del diente. Pero de forma clínica solamente estamos interpretando de forma aproximada, a cuantos milímetros a partir de la CEJ se encuentra la inserción de TC de la encía. También sería necesario calcular la distancia que existe desde la inserción de TC de la encía y el ligamento periodontal hasta el ápice del diente, y esta medida nos representaría el nivel de soporte remanente de un diente (Figura 5). 


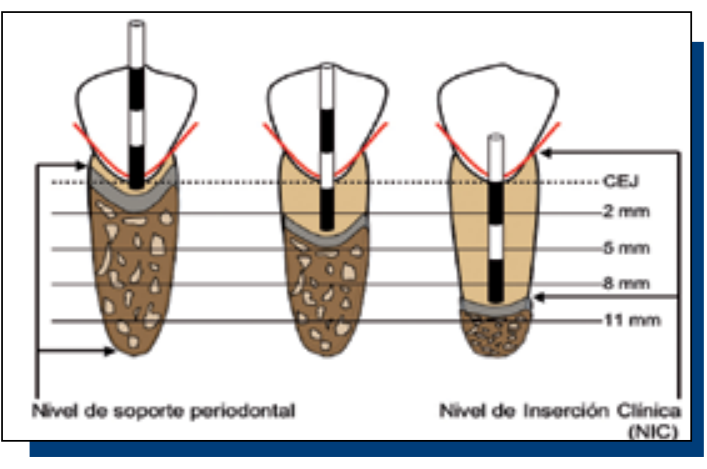

Figura 5. Esquema representativo de la relación entre el nivel de inserción clínica (NIC) y el soporte periodontal.

Para calcular el NIC, se realiza como indica a continuación: - Si el margen esta coronal a la CEJ, se le resta la PS.

- Si el margen coincide con la CEJ, el NIC es igual a la PS.

- Si el margen esta apical a la CEJ, se suma la PS y el margen.

En el ámbito clínico utilizamos el NIC para referirnos a la magnitud de la pérdida de soporte, pero debería ser analizado cuidadosamente en cada diente, ya que es dependiente de la longitud radicular. Por lo tanto, no será lo mismo un NIC de $5 \mathrm{~mm}$ en un canino superior que en un central inferior. Un análisis detallado y cuidadoso diente por diente nos va a mostrar de forma individual el estado aproximado de soporte periodontal.

\section{Sangrado al Sondaje (SS)}

El sangrado al sondaje ha sido uno de los parámetros periodontales más debatidos y analizados ya que se considera que puede ser un predictor de enfermedad periodontal ${ }^{(19,20,21)}$. Pero más que un predictor de enfermedad, puede ser considerado en conjunto con signos clínicos de inflamación, como un indicador de inflamación periodontal. Como el sangrado en este caso es inducido por la penetración de la sonda periodontal, hay que tener en cuenta algunos aspectos del sondaje que pueden hacer variar la interpretación del sangrado al sondaje, como son la fuerza, diámetro de la sonda y grado de inflamación gingival(22). Sería lógico asumir que si sangran durante el sondaje es por que la sonda a llegado hasta el tejido conectivo y en algunos casos, hasta el hueso.

La fuerza es difícil de calcular de forma práctica a menos que se emplee una sonda computarizada (Sonda de Florida) o calibrada. Se ha estimado que una fuerza de $0.75 \mathrm{~N}$ (75 gr/fuerza) con una sonda de $0.63 \mathrm{~mm} \varnothing$ en un periodonto libre de inflamación visible, la sonda se detiene en el epitelio de unión sin llegar al TC. Sin embargo, una persona puede aplicar fuerzas entre $0.15 \mathrm{~N}$ y $0.75 \mathrm{~N}$ y puede que un clínico con suficiente experiencia aplique fuerzas reproducibles cercanas a los $0.75 \mathrm{~N}^{(23,24,25)}$

Pero así se controle la fuerza en cada registro, la sonda puede penetrar más o menos dependiendo del grado de inflamación y diámetro de la sonda. A mayor grado de inflamación gingival, se pierde gradualmente la resistencia de la encía y del EU(26). De igual forma, entre más delgada sea la sonda aún con una fuerza muy ligera, puede penetrar más. Por estas razones es de gran importancia poner gran atención durante el sondaje para evitar errores en la interpretación de los parámetros clínicos periodontales.

De esta forma, el SS debe ser interpretado cuidadosamente y analizado en conjunto con los demás parámetros clínicos ya que su presencia no es un indicativo absoluto de enfermedad (valor predictivo positivo $6 \%$ ) mientras que su ausencia si es un indicador confiable de salud periodontal (valor predictivo negativo 98\%)(27). Para efectos clínicos prácticos, el SS se calcula como el porcentaje de sitios que sangraron al sondaje empleando la formula: SS= sitios que sangran $\times 100 /$ número de dientes $\times 6$.

\section{Línea Mucogingival (LMG)}

La distancia desde el margen gingival hasta la LMG resulta útil para calcular la cantidad de encía queratinizada (EQ) y encía insertada (EI). Se ha estimado que la cantidad de encía aumenta con la edad gracias al proceso de erupción pasiva ${ }^{(28,29)}$. Pero esto solo sería observable en un periodonto que no haya sufrido un trauma significativo durante el cepillado y la masticación, e incluso enfermedad periodontal.
Es necesario diferenciar entre EQ y EI. La EQ es la distancia que hay desde del margen hasta la LMG, mientras que la El es la distancia que hay entre el fondo del surco hasta la LMG. La primera puede ser afectada por la recesión de tejido marginal mientras que la segunda es principalmente afectada por la pérdida de inserción (Figura 6). Ya que esta medida varía de acuerdo al tipo y posición del diente, es importante analizarla cuidadosamente en cada caso. En el ejemplo A (Figura 6 A), se observa una PS mínima y no ha ocurrido recesión de tejido marginal, por lo tanto la encía insertada será igual a la medida de LMG menos la PS. En el ejemplo B es similar (Figura 6 B), solo que ha ocurrido recesión de tejido marginal y ya se ha reducido significativamente tanto la $E Q$ como la El. En contraste, en el ejemplo C (Figura 6 C), la encía aparenta una altura normal pero con una PS incrementada y pérdida de inserción severa. Por lo tanto en este último caso la encía insertada se ha perdido dando como origen la formación de una bolsa periodontal, mientras que la $E Q$ permanece inalterada.

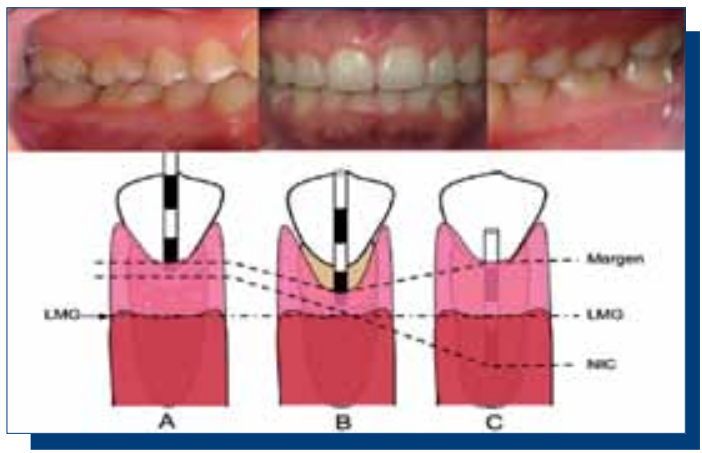

Figura 6. Esquema representativo de las relaciones entre la encía queratinizada, encía insertada y nivel de inserción clínica.

Esto sugiere entonces que tener abundante $E Q$ no es sinónimo de tener abundante El y aunque la encía aparente estar con una altura normal, puede no existir El. Hoy en día sigue en debate la necesidad del aumento del volumen de encía queratinizada y esto queda a consideración del clínico. Sin embargo, en condiciones de higiene oral óptimas, sitios con encía delgada y poca encía queratinizada es posible mantenerlos saludables durante largos períodos de tiempo ${ }^{(30,31,32)}$. La necesidad de aumento de encía queratinizada dependerá de cada caso en particular, evaluando posición dental, presencia de recesiones, higiene oral, necesidades restaurativas, presencia de frenillos sobreinsertados, etc. ${ }^{(33,34)}$.

\section{Movilidad Dental}

Dado que los dientes no están en directo contacto con el hueso alveolar, estos presentan una movilidad fisiológica debido a la presencia del ligamento periodontal. La movilidad dental patológica puede ser el resultado de enfermedad periodontal ${ }^{(35)}$, pero no es la única causa absoluta. El trauma por oclusión ${ }^{(36)}$, ligamentitis y los movimientos ortodónticos, causan movilidad incrementada de los dientes. A diferencia de la movilidad causada por ortodoncia, trauma por oclusión y ligamentitis, la que es causada por periodontitis se incrementa con el tiempo y no es reversible a una movilidad fisiológica. Por lo tanto, es necesario determinar cuidadosamente la causa de la movilidad dental incrementada para resolver el problema.

La movilidad dental se mide de la siguiente forma empleando dos instrumentos metálicos y aplicando presión en sentido vestíbulolingual( ${ }^{(37)}$ :

Grado 0: movilidad fisiológica, 0.1-0.2 mm en dirección horizontal.

Grado 1: movimiento hasta $1 \mathrm{~mm}$ en sentido horizontal.

Grado 2: movimiento de más de $1 \mathrm{~mm}$ en sentido horizontal.

Grado 3: movimiento en sentido horizontal y en sentido vertical.

Es necesario poner especial atención a la movilidad dental patológica, que aumenta progresivamente con el tiempo. Después del tratamiento periodontal, la movilidad se reduce un poco, quedando movilidad residual que puede ser controlada por medio de férulas.

Las medidas periodontales pueden ser registradas en el periodontograma. 
Progresión de la Enfermedad Periodontal (Actividad)

Estudios clínicos longitudinales demostraron que en ausencia de tratamiento periodontal la pérdida anual de inserción y ósea podía estar entre 0.04 y $1.01 \mathrm{~mm}^{(38,39,40)}$. Pero la pérdida de inserción o podía ser sólo atribuida a enfermedad periodontal sino más bien a la suma de varios eventos (masticación, trauma mecánico, cepillado, envejecimiento, etc.). No obstante, nos enfocamos en la identificación de sitios periodontales que pierden inserción por enfermedad periodontal ya que estos representan el punto crítico en el tratamiento periodontal, ya que identificar los sitios en riesgo es todavía un desafío.

Comencemos discutiendo los parámetros que deben analizarse para determinar que hay progresión de la enfermedad. No es posible solamente utilizar un parámetro clínico en particular sino que se debe analizar el conjunto: sangrado al sondaje, profundidad al sondaje y nivel de inserción ${ }^{(41,42)}$. Por consiguiente, en dos examinaciones simultaneas en el tiempo, que si al menos 2 sitios presenten pérdida de inserción proximal de $\geq 3 \mathrm{~mm}$ y con signos de inflamación (SS, PS), serán considerados que presentan "actividad" y pueden seguir perdiendo inserción. Como ayuda adicional, una pérdida ósea en al menos 2 sitios proximales con pérdida ósea de $\geq 2 \mathrm{~mm}$, también será considerado como evidencia de progresión ${ }^{(43)}$. El intervalo de tiempo es variable y depende de cada clínico, pero al menos un intervalo de 4 a 8 semanas entre examinaciones se puede utilizar para estos fines ${ }^{(44)}$. Sin embargo, esto deben contemplarse con mucha cautela ya que clínicamente no es práctico no intervenir al paciente esperando que haya progresión. Pero aún así, las citas de control y mantenimiento durante el tratamiento periodontal nos sirven para identificar sitios en posible riesgo de progresión.

\section{Pérdida Ósea Radiográfica}

Hoy en día sigue siendo un desafío para el desarrollo de la periodoncia tener un sistema suficientemente sensible y de uso rutinario que permita detectar cambios óseos periodontales incipientes (iniciales). Esto dado que el metabolismo óseo es diferente al del tejido conectivo periodontal, evidenciar un cambio significativo requeriría mucho tiempo. Sin embargo, la radiografía periapical nos aporta información importante durante el análisis periodontal como el resultado acumulativo de la enfermedad pasada. Con una secuencia radiográfica en el tiempo, sería posible evaluar los cambios en el nivel óseo. Es importante recordar que uno de los signos más importantes de la periodontitis es la pérdida ósea, la cual debe ser demostrada durante el diagnóstico.

Es necesario buscar cambios radiográficos que están asociados con patología ósea periodontal, como son: pérdida de la continuidad (radiopacidad) de las corticales y crestas óseas, pérdida de la altura ósea y formación de defectos óseos, ensanchamiento del espacio del ligamento periodontal, radiolucidez en zona apical y de furcación. Cabe recordar que la distancia normal de la cresta ósea hasta la CEJ es de $+/-2 \mathrm{~mm}^{(45,46,47)}$.

El patrón de pérdida ósea puede ser horizontal o vertical. La severidad de la pérdida ósea puede ser estimada dividiendo en tercios la distancia desde la CEJ hasta el ápice del diente así: 1/3 cervical (leve), $1 / 3$ medio (moderada) y $1 / 3$ apical (severa) (Figura 7 ).

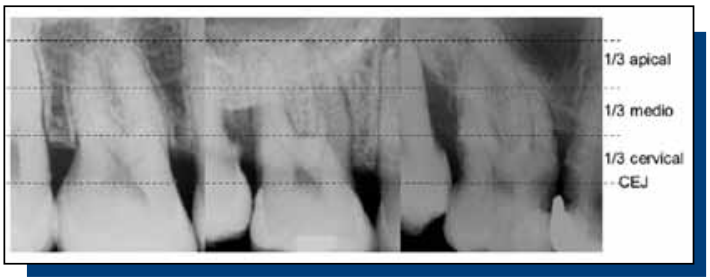

Figura 7. Representación esquemática de los niveles de severidad de pérdida ósea.

Sin embargo, los dientes pueden tener periodonto reducido y no tener lesiones por pérdida ósea. Esto es, la distancia desde la CEJ a la cresta ósea puede aumentar, pero mientras conserve las características radiográficas de salud ósea, será considerado como un periodonto reducido (Figura 8). Este hallazgo es común en pacientes tratados periodontalmente y sujetos de edad avanzada.

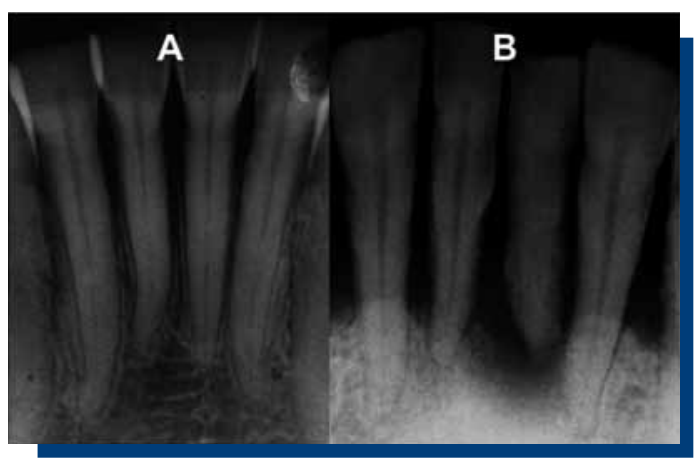

Figura 8. Imágenes radiográficas de (A): periodonto reducido donde se observan crestas alveolares muy delgadas y (B): pérdida ósea severa donde hay pérdida en la continuidad de las corticales y crestas óseas.

La evaluación de la pérdida ósea radiográfica no resulta muy útil como predictor de progresión de enfermedad periodontal en el corto tiempo ${ }^{(48,49)}$. Esto debido a la sensibilidad de la técnica, que no es capaz de identificar cambios mínimos de corto tiempo ${ }^{(50,51,52)}$. Sin embargo, técnicas computarizadas como la radiografía por substracción, permite comparar los niveles de densidad ósea entre dos radiografías ${ }^{(53)}$. Hoy es posible utilizar técnicas radiográficas digitales con software de procesamiento que ayudan a mejorar la imagen, permitiendo hacer una mejor definición de la magnitud de la pérdida ósea.

\section{DIAGNÓSTICO PERIODONTAL}

Es casi universal el uso de la clasificación de la enfermedad periodontal propuesta por la Academia Americana de Periodoncia (AAP, $1999)^{(54,55)}$, pero sin embargo discutiremos algunos aspectos relacionados con el diagnóstico.

Las enfermedades periodontales (gingivitis, periodontitis) que analizaremos a continuación tienen como agente causal principal la placa bacteriana, pero su desarrollo puede ser modificado por condiciones sistémicas (diabetes, discrasias sanguíneas, HIV), hábitos (cigarrillo), estrés y trauma por oclusión.

\section{Gingivitis}

Es la inflamación en diferentes grados de intensidad (Figura 9) de la encía sin afectar los tejidos de soporte (ligamento, cemento, hueso). Los signos de la gingivitis incluyen inflamación y sangrado al sondaje. Aunque puede ocurrir agrandamiento gingival por el edema, dando como resultado el desplazamiento coronal del margen gingival en relación a la CEJ, no existe formación de la bolsa periodontal con pérdida de inserción y hueso. La profundidad al sondaje (hasta $3.9 \mathrm{~mm}$ ) debe ser analizada cuidadosamente para descartar "pseudobolsas periodontales", al igual que la pérdida ósea radiográfica.

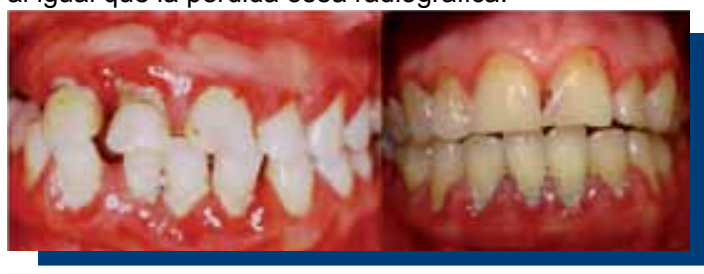

Figura 9. Dos casos de inflamación periodontal con diferentes intensidades.

La parte más difícil es identificar los sitios que ya están evolucionando de gingivitis a periodontitis. Nos podemos encontrar con profundidades de $4 \mathrm{~mm}$ pero que aún no evidencian radiográficamente pérdida ósea y esto es principalmente debido a la baja sensibilidad de la radiografía y al error de sondaje. Se ha calculado que el error al sondaje es de aproximadamente $1 \mathrm{~mm}$ y sumado con el grado de inflamación, fácilmente podemos pasar de $3 \mathrm{~mm}$ a $4 \mathrm{~mm}$ (Figura 10). Esto debe ser analizado cuidadosamente interpretando todos los parámetros clínicos periodontales. 


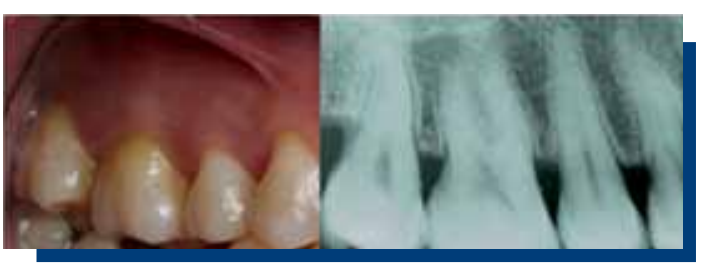

Figura 10. Zona que presenta signos mínimos de inflamación, sangrado al sondaje y profundidades de $3 \mathrm{~mm}$.

La gingivitis no solo aparece en un periodonto con altura normal, sino que puede presentarse en un periodonto con altura reducida y a esto le denominamos "Gingivitis en un periodonto reducido". Adicional a los signos anteriores que son más comunes, también puede aparecer aumento del fluido crevicular incluso llegando al punto de la supuración, movilidad incrementada y dolor.

La extensión de la gingivitis puede ser clasificada como localizada ( $\leq 30 \%$ de sitios afectados) y generalizada $(>30 \%$ de sitios afectados). Asímismo puede ser marginal, papilar y difusa.

\section{Periodontitis Crónica}

A diferencia de la gingivitis, la periodontitis es la inflamación de la encía y el periodonto de soporte, afectando de forma significativa el tejido conectivo gingival (TC), ligamento periodontal, cemento y hueso. Como resultado patognomónico observamos inflamación, sangrado al sondaje (SS), formación de la bolsa periodontal, pérdida de inserción y pérdida ósea radiográfica (Figura 11). Estos signos son mandatorios para realizar el diagnóstico de periodontitis y es una diferencia clara con la gingivitis. Adicionalmente podemos observar recesiones, supuración, movilidad incrementada, migración dental patológica y dolor.

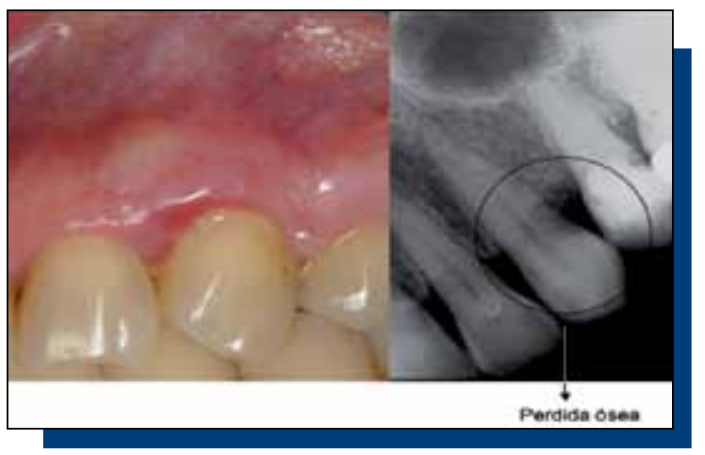

Figura 11. Zona que presenta inflamación, sangrado al sondaje, supuración, pérdida de inserción y profundidad al sondaje de $4 \mathrm{~mm}$ y pérdida ósea radiográfica en un estado inicial.

Resulta problemático cuando no se utilizan criterios de diagnóstico unificados, por esta razón la Federación Europea de Periodoncia (EFP, 2005) ${ }^{(43)}$ define un caso confirmatorio de periodontitis como: $\geq 2$ sitios independientes con pérdida de inserción $\geq 3 \mathrm{~mm}$ proximal y formación de bolsa periodontal. Así mismo, la extensión puede ser clasificada como localizada ( $\leq 30 \%$ de sitios afectados) y generalizada (>30\% de sitios afectados).

En cuanto a la severidad de la destrucción periodontal, el único parámetro que muestra la magnitud del daño, es el nivel de inserción clínica $(\mathrm{NIC})^{(43)}$. Por lo tanto, la severidad de la enfermedad puede ser clasificada como leve, moderada y severa dependiendo del grado de pérdida de inserción en un diente en particular, teniendo como referencia la longitud radicular.

\section{Periodontitis Agresiva}

La periodontitis agresiva se presenta generalmente en sujetos menores de 35 años de edad, pero se sugiere que puede presentarse en cualquier edad. La velocidad de destrucción periodontal es rápida y dado que comienza temprano en la vida, la destrucción se observa en sujetos jóvenes. Pero el análisis no debe basarse solamente en la edad sino en hallazgos clínicos, radiográficos, historia familiar y personal, ayudas de laboratorio.

Las siguientes características primarias están presentes:
1. Aparte de la destrucción periodontal, los pacientes son sistémicamente sanos.

2. Rápida y severa pérdida ósea y de inserción.

3. Agregación familiar.

Algunas características secundarias que generalmente pero no universalmente están presentes:

1. Depósitos microbianos inconsistentes con la destrucción periodontal.

2. Anormalidades fagocíticas.

3. Elevados niveles de Aggregatibacter actinomycetemcomitans y Porphyromonas gingivalis.

4. Elevados niveles de citoquinas inflamatorias (IL-1 $\beta$, PGE2)

5. Hiper-respuesta de macrófagos con alta producción de IL-1 $\beta$.

6. La progresión de la pérdida de inserción y ósea puede detenerse por si sola.

Las periodontitis agresivas se han clasificado en dos formas clínicas de la siguiente manera: periodontitis agresiva localizada que presenta (1) establecimiento durante la pubertad y (2) afecta incisivos y primeros molares; y periodontitis agresiva generalizada presentando (1) afección de sujetos menores de 30 años, pero se puede presentar en sujetos mayores y (2) aparte de incisivos y primeros molares, afecta más de 3 dientes adicionales.

Respecto de las periodontitis agresivas, sigue siendo un desafío llegar a un consenso en la comunidad científica en la forma como se evalúan las características enunciadas anteriormente. Es importante resaltar que los signos clínicos determinantes de una periodontitis (bolsa periodontal, inflamación y sangrado, pérdida ósea) están presentes y no son significativamente diferentes a los de una periodontitis crónica. La composición microbiana ya se demostró no determina el diagnóstico ${ }^{(56)} y$ las pruebas sobre la respuesta inmunológica todavía no son definitivas y practicas desde el punto de vista clínico. Adicionalmente, con excepción del uso de antibióticos sistémicos, el tratamiento periodontal sigue siendo el mismo para ambos tipos de periodontitis. Entonces, ¿qué es la periodontitis agresiva? Es una pregunta que aún necesita de evidencia científica consistente. Por tanto, si se hace un análisis detallado del termino empleado, "agresiva" implica que es más destructiva que la periodontitis crónica. Esto quiere decir que durante el análisis clínico y en presencia de un posible caso de periodontitis agresiva, se debería calcular la velocidad de destrucción periodontal en un tiempo determinado sin hacer tratamiento al paciente. Pero esto no resulta ni ético ni práctico desde la perspectiva clínica. Sin embargo, un cuidadoso análisis de la expresión clínica de la enfermedad, considerando variables periodontales locales y variables del sujeto (Ej.: agregación familiar), será posible determinar si en realidad nos estamos enfrentando a un caso en donde la destrucción periodontal pueda avanzar a una velocidad mucho mayor que la periodontitis crónica si no se aplica el tratamiento adecuado. Revisiones de la literatura recientes sugieren que en la actualidad, no existen diferencias definitivas entre los signos y la expresión clínica, así como las características inmunológicas que permitan en conjunto establecer una diferencia etiopatológica entre periodontitis agresiva y periodontitis crónica ${ }^{(57,58,59)}$. Más aún, la respuesta al tratamiento periodontal y el mantenimiento de la salud periodontal es muy similar entre ambas patologías ${ }^{(60)}$.

\section{CONCLUSIONES}

La expresión clínica de la enfermedad periodontal involucra una serie de alteraciones en los tejidos periodontales. La interpretación y medición correcta de las variables clínicas nos permiten determinar un diagnóstico correcto y por lo tanto, un tratamiento exitoso. Aún así, la expresión clínica de la enfermedad no es igual en todos los pacientes y por lo tanto el análisis debe ser individual para cada caso. 


\section{REFERENCIAS BIBLIOGRÁFICAS}

1. Dale BA. Periodontal epithelium: a newly recognized role in health and disease. Periodontol,2000,2002;30:70-78.

2. Bosshardt DD, Lang $\mathrm{N}$. The junctional epithelium: from health to disease. $J$ Dent Res,2005;84:9-20.

3. Nanci A, Bosshardt DD. Structure of periodontal tissues in health and disease. Periodontol,2000,2006;40:11-28.

4. Choi EY, Santoso S, Chavakis T. Mechanisms of neutrophil transendothelial migration. Front Biosci,2009 1;14:1596-605.

5. Kornman KS, Page RC, Tonetti MS. The host response to the microbial challenge in periodontitis: assembling the players. Periodontol,2000,1997;14:33-53.

6. Abbas AK, Lichtman AH, Pillai S. Effector mechanisms of cell mediated immunity. In: Cellular and molecular immunology. Saunders Elsevier $6^{\text {th }}$ edition Philadelphia, PA, 2007;303-320.

7. Page RC, Schroeder HE. Pathogenesis of inflammatory periodontal disease. A summary of current work. Lab Invest,1976;34:235-249.

8. Weinberg MA, Eskow RN. Periodontal terminology revisited. J Periodontol, 2003;74:563-565

9. Caton J, Greenstein G, Polson AM. Depth of periodontal probe penetration related to clinical and histologic signs of gingival inflammation. J Periodontol, 1981;52:626-629.

10. Müller HP, Eger T. Masticatory mucosa and periodontal phenotype: a review. Int J Periodontics Restorative Dent,2002;22:172-183.

11. Novak MJ, Albather HM, Close JM. Redefining the biologic width in severe, generalized, chronic periodontitis: implications for therapy. J Periodontol, 2008;79:1864-189.

12. Greenstein G. Current interpretations of periodontal probing evaluations: diagnostic and therapeutic implications. Compend Contin Educ Dent, 2005;26:381-390

13. Gargiulo AW, Wentz FM, Orban B. Dimensions and relations of the dentogingival junction in humans. J Periodontol,1961;32:261-267.

14. Vacek JS, Gher ME, Assad DA, Richardson AC, Giambarresi LI. The dimensions of the human dentogingival junction. Int J Periodontics Restorative Dent, 1994;14:154-165.

15. Toto PD, Gargiulo AW. Epithelial and connective tissue changes in periodontitis. J Periodontol,1970;41:587-590.

16. Listgarten MA. Periodontal probing: what does it mean? J Clin Periodontol, 1980;7:165-176

17. Matuliene G, Pjetursson BE, Salvi GE, Schmidlin K, Brägger U, Zwahlen M, Lang NP. Influence of residual pockets on progression of periodontitis and tooth loss: results after 11 years of maintenance. J Clin Periodontol,2008;35:685-695. 18. Brägger U, Nyman S, Lang NP, von Wyttenbach T, Salvi G, Schürch E Jr. The significance of alveolar bone in periodontal disease. A long-term observation in patients with cleft lip, alveolus and palate. J Clin Periodontol,1990;17:379-384. 19. Joss A, Adler R, Lang NP. Bleeding on probing. A parameter for monitoring periodontal conditions in clinical practice. J Clin Periodontol,1994;21:402-408.

20. Lang NP, Joss A, Tonetti MS. Monitoring disease during supportive periodontal treatment by bleeding on probing. Periodontol,2000,1996;12:44-48. 21. Lang NP, Joss A, Orsanic T, Gusberti FA, Siegrist BE. Bleeding on probing. A predictor for the progression of periodiontal disease? J Clin Periodontol, 1986;13:590-596.

22. Lang NP, Nyman S, Senn C, Joss A. Bleeding on probing as it relates to probing pressure and gingival health. J Clin Periodontol,1991;18:257-261.

23. van der Velden U. Influence of periodontal health on probing depth and bleeding tendency. J Clin Periodontol,1980;7:129-139.

24. van der Velden $U$, Jansen J. Probing forces in relation top robe penetration into the periodontal tissues in dogs. A microscopic evaluation. J Clin Periodontol, 1980;7:325-327

25. van der Velden U, Jansen J. Microscopic evaluation of pocket depth measurements performed with six different probing forces in dogs. $J$ Clin Periodontol,1981;8:107-116

26. Armitage GC, Svanberg GK, Löe H. Microscopic evaluation of clinical measurements of connective tissue attachment levels. J Clin Periodontol, 1977:4:173-190.

27. Lang NP, Adler R, Joss A, Nyman S. Absence of bleeding on probing. An indicator of periodontal stability. J Clin Periodontol,1990;17:714-21.

28. Talari A, Ainamo J. Orthopantomographic assessment of the width of attached gingiva. J Periodontal Res,1976;11:177-181.

29. Ainamo J, Talari A. The increase with age of the width of attached gingiva. $J$ Periodontal Res,1976;11:182-188.

30. Lang NP, Löe $H$. The relationship between the width of keratinized gingiva and gingival health. J Periodontol,1972;43:623-627.

31. Miyasato $M$, Crigger M, Egelberg J. Gingival condition in areas of minimal and appreciable width of keratinized gingiva. J Clin Periodontol,1977;4:200-209.
32. Wennström J, Lindhe J, Nyman S. The role of keratinized gingiva in plaqueassociated gingivitis in dogs. J Clin Periodontol,1982;9:75-85.

33. Oh SL. Attached gingiva: histology and surgical augmentation. Gen Dent, 2009;57:381-385.

34. Agudio G, Nieri M, Rotundo R, Franceschi D, Cortellini P, Pini Prato GP Periodontal conditions of sites treated with gingival-augmentation surgery compared to untreated contralateral homologous sites: a 10- to 27-year longterm study. J Periodontol,2009;80:1399-1405.

35. Goodson JM. Diagnosis of periodontitis by physical measurement: interpretation from episodic disease hypothesis. J Periodontol,1992;63(4 Suppl):373-382.

36. Ruiz JL. Seven signs and symptoms of occlusal disease: the key to an easy diagnosis. Dent Today,2009;28:112-113.

37. Salvi GE, Lindhe J, Lang NP. Examination of patients with periodontal disease. In: Lindhe J. Lang NP, Karring T. Clinical periodontology and implant dentistry. Blackwell-Munksgaard $5^{\text {th }}$ edition, Oxford UK. 2008: 573-586.

38. Löe H, Anerud A, Boysen H, Morrison E. Natural history of periodontal disease in man. Rapid, moderate and no loss of attachment in Sri Lankan laborers 14 to 46 years of age. J Clin Periodontol,1986;13:431-445.

39. Baelum V, Luan WM, Chen X, Fejerskov O. A 10-year study of the progression of destructive periodontal disease in adult and elderly Chinese. $J$ Periodontol, 1997:68:1033-1042

40. Baelum V, Luan WM, Chen X, Fejerskov O. Predictors of destructive periodontal disease incidence and progression in adult and elderly Chinese. Community Dent Oral Epidemiol,1997;25:265-272.

41. Halazonetis TD, Haffajee AD, Socransky SS. Relationship of clinical parameters to attachment loss in subsets of subjects with destructive periodontal diseases. J Clin Periodontol,1989;16:563-568.

42. Haffajee AD, Socransky SS, Lindhe J, Kent RL, Okamoto H, Yoneyama T. Clinical risk indicators for periodontal attachment loss. J Clin Periodontol, 1991;18:117-125.

43. Tonetti MS, Claffey N, on behalf of the European Workshop in Periodontology group C. Advances in the progression of periodontitis and proposal of definitions of a periodontitis case and disease progression for use in risk factor research. $J$ Clin Periodontol,2005;32 (Suppl. 6):205-208.

44. Gamonal J, BasconesA, Jorge O, SilvaA: Chemokine RANTES in gingival crevicular fluid of adult patients with periodontitis. J Clin Periodontol,2000;27:675-681.

45. Regan JE, Mitchell DF. Roentgenographic and dissection measurements of alveolar crest height. J Am Dent Assoc,1963;66:356-359.

46. Källestål $C$, Matsson L. Criteria for assessment of interproximal bone loss on bite-wing radiographs in adolescents. J Clin Periodontol,1989;16:300-304.

47. Hausmann E, Allen K, Clerehugh V. What alveolar crest level on a bite-wing radiograph represents bone loss? J Periodontol, 1991;62:570-572.

48. Khocht A, Zohn H, Deasy M, Chang KM. Screening for periodontal disease: radiographs vs. PSR. J Am Dent Assoc, 1996;127:749-756.

49. Reddy MS. Radiographic alveolar bone change as an outcome measure for therapies that inhibit bone loss or foster bone gain. $J$ Int Acad Periodontol, 2005;7(4 Suppl):175-188.

50. Brägger U, Håkanson D, Lang NP. Progression of periodontal disease in patients with mild to moderate adult periodontitis. J Clin Periodontol,1992;19:659-666.

51. Jeffcoat MK. Radiographic methods for the detection of progressive alveolar bone loss. J Periodontol,1992;63(4 Suppl):367-372.

52. Jeffcoat MK, Reddy MS. A comparison of probing and radiographic methods for detection of periodontal disease progression. Curr Opin Dent,1991;1:45-51.

53. Nummikoski PV, Steffensen B, Hamilton K, Dove SB. Clinical validation of a new subtraction radiography technique for periodontal bone loss detection. $J$ Periodontol,2000;71:598-605.

54. Armitage GC. Development of a classification system for periodontal diseases and conditions. Ann Periodontol,1999;4:1-6.

55. Armitage GC. Periodontal diagnoses and classification of periodontal diseases. Periodontol,2000,2004;34:9-21.

56. Mombelli A, Casagni F, Madianos PN. Can presence or absence of periodontal pathogens distinguish between subjects with chronic and aggressive periodontitis? A systematic review. J Clin Periodontol,2002;29 (Suppl 3):10-21.

57. Armitage GC, Cullinan MP. Comparison of the clinical features of chronic and aggressive periodontitis. Periodontol,2000,2010;53:12-27.

58. Smith M, Seymour GJ, Cullinan MP. Histopathological features of chronic and aggressive periodontitis. Periodontol,2000,2010;53:45-54.

59. Ford PJ, Gamonal J, Seymour GJ. Immunological differences and similarities between chronic periodontitis and aggressive periodontitis. Periodontol,2000,2010 Jun;53:111-23.

60. Deas DE, Mealey BL. Response of chronic and aggressive periodontitis to treatment. Periodontol,2000,2010;53:154-66. 